\title{
SENTINEL-1 PRECISE ORBIT CALIBRATION AND VALIDATION
}

\author{
Andrea Monti Guarnieri, Simone Mancon, and Stefano Tebaldini \\ Politecnico di Milano, Italy
}

\begin{abstract}
In this paper, we propose a model-based procedure to calibrate and validate Sentinel-1 orbit products by the Multi-Squint (MS) phase. The technique allows to calibrate an interferometric pair geometry by refining the slave orbit with reference to the orbit of a master image. Accordingly, we state the geometric model of the InSAR phase as function of positioning errors of targets and slave track; and the MS phase model as derivative of the InSAR phase geometric model with respect to the squint angle. In this paper we focus on the TOPSAR acquisition modes of Sentinel-1 (IW and EW) assuming at the most a linear error in the known slave trajectory. In particular, we describe a dedicated methodology to prevent InSAR phase artifacts on data acquired by the TOPSAR acquisition mode. Experimental results obtained by interferometric pairs acquired by Sentinel-1 sensor will be displayed.
\end{abstract}

Key words: Sentinel1; InSAR; orbit calibration; MS phase.

\section{INTRODUCTION}

Sentinels Precise Orbit Determination (POD) service provides orbit products containing satellite state vectors with the accuracy of some centimeters [11]. Still, the InSAR phase is very sensitive even to small orbital error (a few millimeters). The problem of the baseline error has already been addressed in literature concerning airborne SAR systems [7], although the authors just estimate the derivatives of baseline errors treating the mis-registration as an error to remove, and they do not take into account strong errors in Digital Elevation Model (DEM) knowledge. Concerning spaceborne SAR systems, [5] describes InSAR phase artifacts due to orbital errors on RADARSAT and calibrates orbit information by an interferometric stack. However, this method is unable to separate phase contribution due to orbit from APS. In fact, [1] explains that retrieving geometrical parameters from InSAR phase (spaceborne case) is important to take into account all the following contributions: the Atmospheric Phase Screen (APS), the DEM errors and orbit errors. In this paper we exploit the MS analysis to estimate the ac- curacy of a sensor's state vectors and to calibrate InSAR phase, with the key assumption that APS and DEM error contributions can be neglected from MS phase [4]. It is important to point out that MS phase (spectral diversity technique) has already been used in some works in order to calibrate interferometric pairs of space-borne systems $[6,9]$. However, they use MS phase to estimate baseline error in along-track direction, whereas, we derive a complete theoretical model and evaluate the MS phase sensitivity to each component. The precise estimation of the model parameters provides both an qualification of the orbit accuracy and a residual phase screen that can be used for removing systematic phase errors in repeat-pass interferograms, hence for phase validation. The approach here proposed is quite suited to Sentinel-1 mission thanks to (1) the large swath and (2) the large squint diversity in TOPSAR mode. In fact, TOPSAR data are more sensitive to orbit errors due to high Doppler centroid variation and non-continuous acquisition. For example, if alongtrack mis-registration affects squinted stripmap interferometry just as a phase constant, it becomes a phase ramp for TOPSAR interferometry, because the Doppler centroid changes fast inside bursts. Furthermore it generates phase jumps in bursts transition where Doppler centriod is discontinuous [6].

\section{GEOMETRIC ERROR IN INSAR PHASE}

The geometric contribution to InSAR phase of each targets, depends on three vectors, $\mathbf{S}_{\mathbf{1}}, \mathbf{S}_{\mathbf{2}}$ and $\mathbf{P}$, see Fig. 1.

$$
\Delta \phi=\frac{4 \pi}{\lambda}[\Delta R]=\frac{4 \pi}{\lambda}\left[\left|\mathbf{S}_{\mathbf{2}}-\mathbf{P}\right|-\left|\mathbf{S}_{\mathbf{1}}-\mathbf{P}\right|\right]
$$

where $\mathbf{S}_{\mathbf{1}}$ and $\mathbf{S}_{\mathbf{2}}$ define sensor positions on master and slave tracks respectively and $\mathbf{P}$ is the target position. For the sake of simplicity we define hereinafter just the model for difference of distances between target and trajectories of two interferometric acquisitions. The theoretical model of InSAR phase can be obtained from relationships expressed in distances just multiplying to $\frac{4 \pi}{\lambda}$. In the interferometric processing chain, the flattening is a very common step which removes the contribution of known geometry [8]. After this step some phase artifacts may affect the interferogram, if the reference DEM and known state vectors differ from the actual geometry. In partic- 


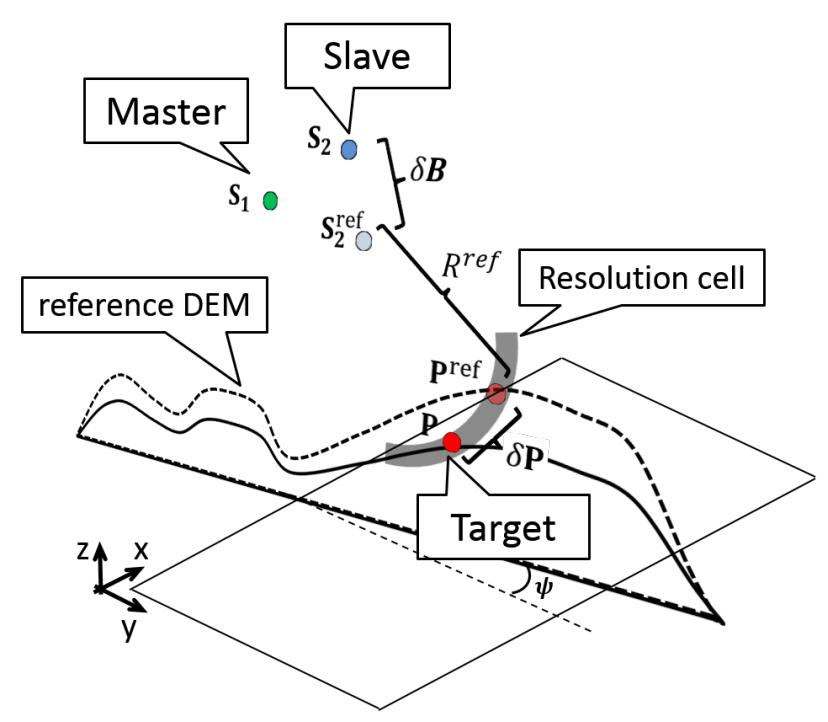

Figure 1: Geometric error components on the plane defined by the squint used during the focusing step.

ular, interferometry senses difference of distances, therefore we report in (2) the geometric error $\Delta R_{e r r}$ as the difference between master and slave errors $\left(\Delta R_{e r r, 1}\right.$ and $\left.\Delta R_{e r r, 2}\right)$.

$$
\begin{array}{r}
\Delta R_{e r r}=\Delta R_{e r r, 2}-\Delta R_{\text {err }, 1}= \\
\left|\mathbf{S}_{\mathbf{2}}-\mathbf{P}\right|-\left|\mathbf{S}_{\mathbf{2}}^{\text {ref }}-\mathbf{P}^{\text {ref }}\right|+ \\
-\left|\mathbf{S}_{\mathbf{1}}-\mathbf{P}\right|+\left|\mathbf{S}_{\mathbf{1}}^{\text {ref }}-\mathbf{P}^{\text {ref }}\right|
\end{array}
$$

Where $\mathbf{S}_{1}^{\text {ref }}, \mathbf{S}_{2}^{\text {ref }}$ and $\mathbf{P}^{\text {ref }}$ are respectively the known vector position of master, slave and target. As mentioned, this method aims to calibrate interferometric pairs, hence we can assume to know the distance between master track and current target position $\left(\Delta R_{e r r, 1}=0\right)$. According to this statement geometrical errors can be defined as follows:

$$
\Delta R_{e r r}=\left|\mathbf{S}_{\mathbf{2}}-\mathbf{P}\right|-\left|\mathbf{S}_{\mathbf{2}}^{\text {ref }}-\mathbf{P}^{\text {ref }}\right|
$$

The geometric error is displayed in Fig. 1 as sum of two vectors : $\delta \mathbf{B}=\left[\delta B_{x}, \delta B_{y}, \delta B_{z}\right]$ and $\delta \mathbf{P}=[\delta x, \delta y, \delta z]$. Where $\delta \mathbf{B}$ is the distance between known and actual track of slave image and $\delta \mathbf{P}$ the difference between the reference DEM (i.e. the DEM used for interferogram flattening) and the actual DEM. Fig. 1 displays the two vector components of the geometric error on a plane defined by the current target position and the squint angle used in focusing $(\psi)$. The InSAR phase error, derived from (3), depends just on the errors in slave track and target position. This is not true in general but we can anyway calibrate a interferometric stack (w.r.t. a master image) assuming geometric errors affect just tracks of slaves acquisitions. In Equation (4) we report the difference distance error depending on the known geometry (squint and look angles).

$$
\begin{array}{r}
\Delta R_{e r r}\left(\psi, \theta_{S 1}, \theta_{S 2}\right)=\sin \psi \cdot \delta B_{x}\left(\tau_{\psi}\right)+ \\
-\cos \psi \cdot \sin \theta_{S 2} \cdot \delta B_{y}\left(\tau_{\psi}\right)+ \\
+\cos \psi \cdot \cos \theta_{S 2} \cdot \delta B_{z}\left(\tau_{\psi}\right)+ \\
+\cos \psi \cdot\left(\sin \theta_{S 2} \cdot \cot \theta_{S 1}-\cos \theta_{S 2}\right) \cdot \delta z,
\end{array}
$$

where $\theta_{S 1}$ and $\theta_{S 2}$ are the look angles of master and slave image respectively and $\psi$ is the squint angle. We assume that the interferometric pair is composed by images with almost the same squint angle, otherwise an azimuth common-band filtering is assumed in (4). It is important pointing out that in (4) we keep just one DEM error component $(\delta z)$. This is because we can assume to know the range distance between target and sensor and the squint angle used by the focusing step. Therefore the vector $\delta P$ is forced to lie on a circumference, as shown in Fig. 1, and we can characterize it as a one-dimensional variable (i.e. the elevation angle direction).

\section{GEOMETRIC ERROR IN MS PHASE}

The relationship (4) defines the components of the InSAR phase due to geometric error. However, InSAR phase is, in general, influenced by others components (orbit, DEM, SAR sensor, APS, etc..) and it is difficult to separate different contributions. For this reason we exploit the MultiSquint (MS) phase, which mainly depends on orbit errors. Assuming a linear orbit error:

$$
\delta \mathbf{B}(\tau)=\frac{1}{\Delta \tau} \int_{\Delta \tau} \delta \mathbf{B}(\tau) d \tau+\tau \frac{\partial \delta \mathbf{B}}{\partial \tau}=\delta \overline{\mathbf{B}}+\tau \cdot \Delta \delta \mathbf{B}
$$

we can write the theoretical model for the MS phase as the relationship (6), i.e. as derivative of (4) with respect to the squint angle, see Fig. 2.

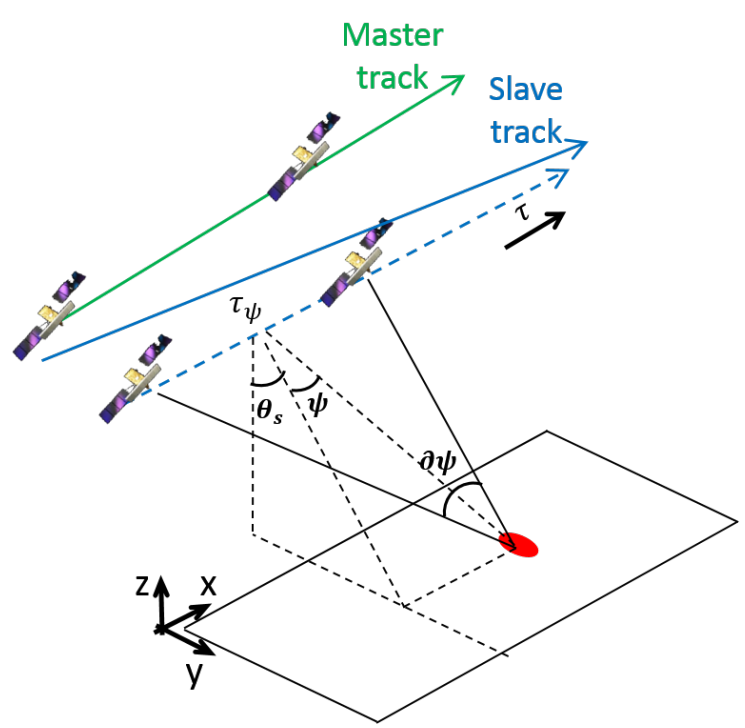

Figure 2: 3D sketch of a squinted acquisition geometry for MS InSAR analysis.

$$
\begin{array}{r}
\frac{\partial \Delta R_{e r r}}{\partial \psi}=\cos \psi \cdot \delta \bar{B}_{x}+ \\
+\sin \psi \cdot R_{0} \cdot \Delta \delta B_{x}+ \\
+\sin \psi \cdot \sin \theta_{S} \cdot \delta \bar{B}_{y}+ \\
-\sin \psi \cdot \cos \theta_{S} \cdot \delta \bar{B}_{z}+
\end{array}
$$




$$
\begin{array}{r}
-\cos \psi \cdot \sin \theta_{S} \cdot R_{0} \cdot \Delta \delta B_{y}+ \\
+\cos \psi \cdot \cos \theta_{S} \cdot R_{0} \cdot \Delta \delta B_{z}+ \\
+\sin \psi \cdot\left(\sin \theta_{S 2} \cdot \cot \theta_{S 1}-\cos \theta_{S 2}\right) \cdot \delta z
\end{array}
$$

where $R_{0}$ is the reference distance between the current target and the slave trajectory. The Equation (6) defines a pixelwise model which aims to generalize the MS phase behavior with reference to known and unknown geometric parameters. For the sake of the clearness we can approximate the derivative of InSAR phase with respect to the squint angle as the difference between two azimuth sub-apertures. Indeed, the correspondence between azimuth frequencies and squint angles allows to separate data components received by different squint angles.

\section{REDUCING RANK OF PARAMETRIZATION}

In this section we investigate the sensitivity of the linearized model defined in Eq. 6 to each unknown, to define how many parameters we can estimate with millimetric accuracy. First of all is necessary to convert (6) in the following matrix equation:

$$
\mathbf{F} \cdot \boldsymbol{\Omega}=\phi_{m s}
$$

Where $\mathbf{F}$ corresponds to the matrix of the linearized problem (6) and $\Omega$ the vector of the geometric errors (8) (the parameters to estimate).

$$
\boldsymbol{\Omega}=\left[\delta \bar{B}_{x}, \delta \bar{B}_{y}, \delta \bar{B}_{z}, \Delta \delta B_{x}, \Delta \delta B_{y}, \Delta \delta B_{z}, \delta z\right]
$$

and $\phi_{m s}$ the observable (i.e. the MS phase). In order to evaluate how much each unknown influences the MS phase we perform the singular values analysis of the $\mathbf{F}$ matrix in case of IW and EW acquisition modes.

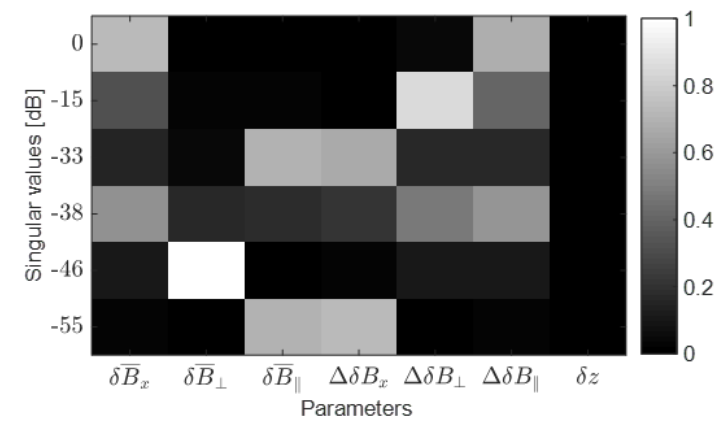

Figure 3: Singular values analysis of linearized problem (6) for a typical TOPSAR IW swath.

In Figures 3 and 4 we display the matrix of right-singular vectors of the forward problem (from SVD analysis of $\mathbf{F}$ matrix). On the vertical axis of these we annotate the relative amplitude of singular values in $\mathrm{dB}$ (normalized to the highest singular value), whereas on the horizontal axis we have the unknowns. Each element of the matrix contains,

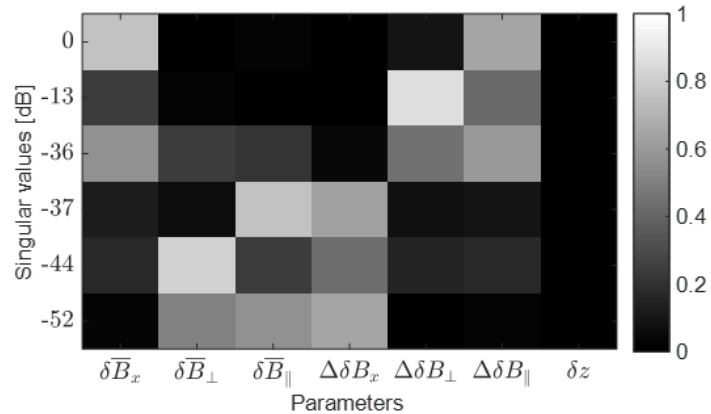

Figure 4: Singular values analysis of linearized problem (6) for a typical TOPSAR EW swath.

therefore, the normalized projection of data vector due to the current unknown (identified by current column) on the right-singular vector (identified by current row). This kind of visualization allows to understand how the linear system amplifies each unknown, i.e. it shows the MS phase's sensitivity to each of them. Therefore we redefined the theoretical model selecting just two of the unknowns with energy in first singular values. This simplification allows to mitigate the noise effects and achieving the requested accuracy computed by the following formula:

$$
\left.\sigma_{\boldsymbol{\Omega}}^{2}=\operatorname{diag}\left(\left(\mathbf{F}^{H} \mathbf{F}\right)^{-1} \cdot \mathbf{F}^{H}\left(\sigma_{\text {res }}^{2} I\right) \mathbf{F}(\mathbf{F F})^{H}\right)^{-1}\right)
$$

Where we approximate the variance of the observable $\left(\sigma_{\mathbf{w}}^{2}\right)$ as the variance of the residue $\left(\sigma_{\text {res }}^{2}\right)$. Each element of the $\sigma_{\Omega}^{2}$ vector corresponds to the variance of the geometric errors estimated and $\operatorname{diag}(\cdot)$ operator is the operator which returns the diagonal elements of a matrix. Accordingly, we redefine the linearized problem in Equation (6) keeping just two geometric unknowns with energy in the first two singular values, and the result is the simplified linear problem stated in Equation (10).

$$
\begin{array}{r}
\frac{\partial \Delta R_{e r r}}{\partial \psi}=\cos \psi \cdot \delta \bar{B}_{x}+ \\
-\cos \psi \cdot \sin \left(\theta_{S}-\theta_{r e f}\right) \cdot R_{0} \cdot \Delta \delta B_{\perp}+
\end{array}
$$

We use this simplified model in the estimation algorithm described in the next section, and $\boldsymbol{\Omega}_{s}=\left[\delta \bar{B}_{x}, \Delta \delta B_{\perp}\right]$ is the new vector of unknowns.

\section{NON-LINEAR ESTIMATION OF BASELINE ERROR}

In this section we describe the suggested methodology to estimate the orbit error components by MS phase. The flowchart of the processing chain is shown in Fig. 5 and described hereinafter. Consider an inteferomentric pair focused and coregistered by geometric information. We emphasize that coregistration procedure take into account just geometric information (i.e. reference DEM, master and slave state vectors). Even more, assume each 


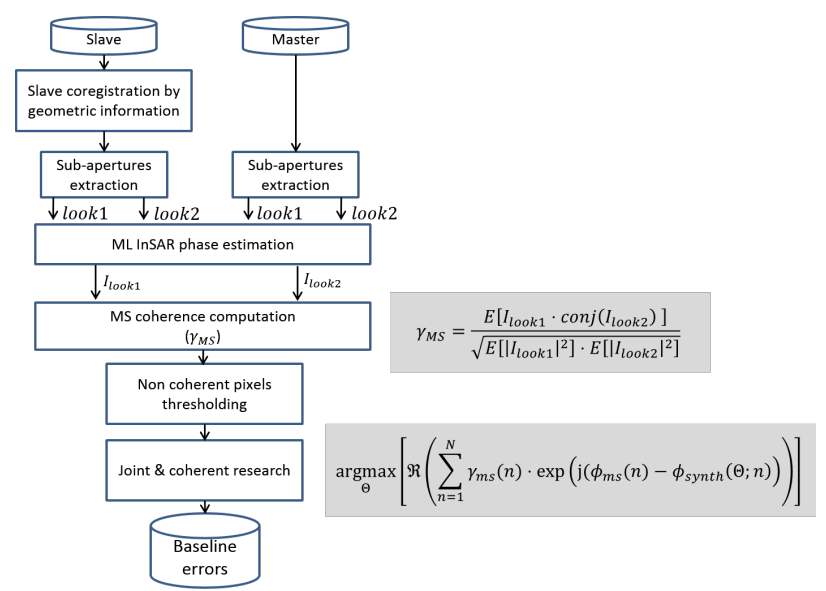

Figure 5: Flowchart of the suggested technique.

coregistered SLC is splitted in two sub-apertures nonoverlapping in azimuth frequency. Concerning TOPSAR acquisition modes, sub-apertures can be obtained exploiting portion of data acquired by two different central azimuth frequencies (burst and swath overlaps)[6]. In the first step of algorithm we exploit the Maximum Likelihood (ML) estimator of the InSAR phase described in [3]. Assuming the SLC data as a random variable distributed as a circular Gaussian distribution, the multilooked interferogram is the ML estimator of the interferometric phase ( $I_{l o o k 1}$ and $I_{l o o k 2}$ are the two multi-looked interferograms). As result of this step we have an estimation of interferometric phase for each sub-aperture $\left(\Delta \phi_{k 1}=\angle\left(I_{l o o k 1}\right)\right.$ and $\left.\Delta \phi_{k 2}=\angle\left(I_{l o o k 2}\right)\right)$. In the second step we estimate the MS phase as difference between the two InSAR phase computed at the previous step $\left(\phi_{m s}=\Delta \phi_{k 2}-\Delta \phi_{k 1}\right)$. According to our experience it is very useful assigning a quality measure to each MS phase value, in order to perform a weighted estimation. A good measure of the MS phase quality is a sort of coherence between the two interferograms.

$$
\gamma_{m s}=\frac{E\left[I_{l o o k 1} \cdot \operatorname{conj}\left(I_{l o o k 2}\right)\right]}{\sqrt{E\left[\left|I_{l o o k 1}\right|^{2}\right] \cdot E\left[\left|I_{l o o k 2}\right|^{2}\right]}}
$$

We will name this quality index "MS coherence" $\left(\gamma_{m s}\right)$, and we define it in Equation (11). As is displayed in Fig. 5 , we apply here a threshold on the "MS coherence" discarding the measures with low reliability. In the last step we intend to estimate baseline errors using the MS phase and the parametric model defined in Section 4. We suggest an exhaustive research of the geometric parameters set which best explain the MS phase just computed. In order to do this we defined the minimization problem as follows:

$$
\arg \max _{\Theta}\left\{\Re\left(\sum_{n=1}^{N} \gamma_{m s}(n) \cdot e^{j\left(\phi_{m s}(n)-\phi_{s y}\left(\Omega_{s} ; n\right)\right)}\right)\right\}
$$

Where $\phi_{s y}$ is the synthetic MS phase computed according to the Equation (10), $N$ is the number of measures of the
MS phase used for the estimation and $\Omega_{s}$ is the set of all values possible for the couple of geometric parameters in Equation (10).

\section{EXPERIMENTAL RESULTS}

In this section we report the results of MS analysis applied to interferometric pairs acquired by Sentinel-1 satellite. In order to do this, we first of all have performed the coregistration using the state vectors provided and the DEM Srtm, at a later stage we performed the MS analysis. The results of MS phase analyses have been collected for: "Restituted Orbit State Vectors" (RESORB) and "Precise Orbit Ephemerides" (POEORB). We refer to [10] for more details about Sentinel-1 orbit products. Concerning the restituted and precise orbits we report hereinafter the results of MS analysis in Fig. 6. The results reported confirm the expected accuracy for both "Restituted Orbit State Vectors" product (less than $10 \mathrm{~cm}$ ) and the higher accuracy of "Precise Orbit Ephemerides" $(5 \mathrm{~cm})$ [10]. In accordance with the orbit accuracy mea-

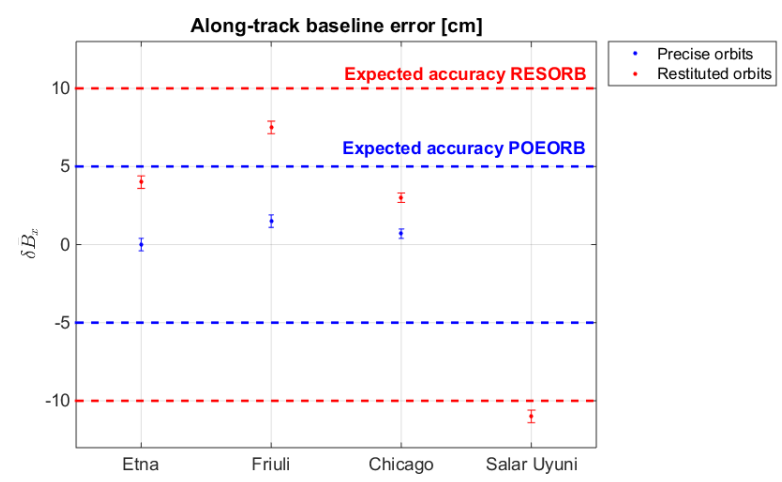

Figure 6: Sentinel-1 orbit errors estimation for restituted and precise orbit products. The error-bar of each measure defines the accuracy of the estimation.

sured by the technique on "Precise Orbit Ephemerides", we obtain a very good interferogram (free of artifacts due to orbits errors) over Etna volcano. A portion of IW interferogram mentioned above is available in Fig. 9. In general even the restituted orbits accuracy is enough to prevent InSAR phase artifacts. We just display in Fig. 7 a particular test case where the MS analysis allow to produce a good interferogram free of artifacts. In order to show the order of magnitude of the InSAR phase error estimated Fig. 8 reports the synthetic InSAR fringes removed.

A particular attention is needed for non-stationary areas, in fact if targets in the scene change their position in the lapse of time which separates the interferometric pair, the MS phase senses this motion. In particular this happens if the motion vector has component in along-track direction. Accordingly, we do not report the geometric error estimated over San Francisco because an earthquake hits 


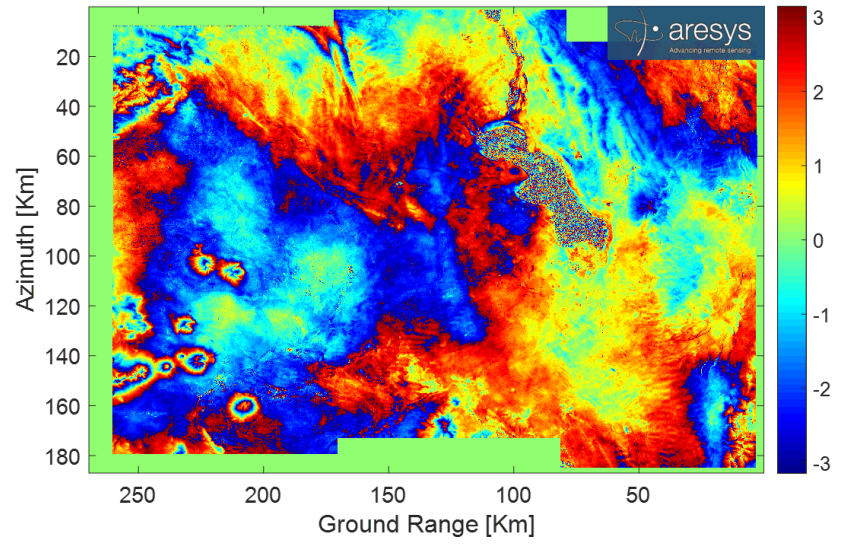

Figure 7: Salar Uyuni: Flattened interferogram, coregistration has been performed by "Restituted orbit" product refined by the MS analysis.

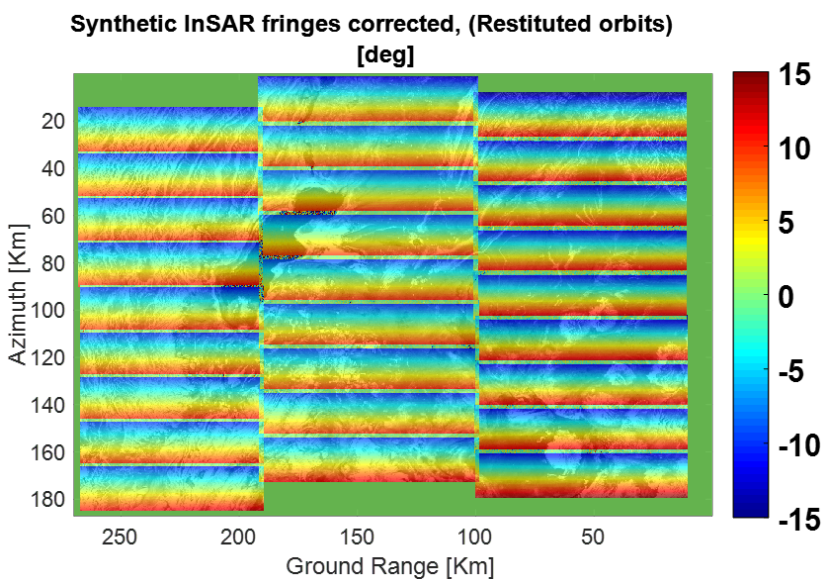

Figure 8: Salar Uyuni: synthetic InSAR fringes due to baseline error estimated for "Restituted orbit" product. The residual fringes are superimposed on the absolute value of the interferogram.

this area in the period which separates the two acquisitions. Therefore, the estimation is probably biased by the earth motion. We just report in Fig. 10 both InSAR phase and MS phase which respectively sense the line-of-sight and along-track motion components [2].

\section{CONCLUSIONS}

The suggested technique has been demonstrated as a valuable tool for interferometric pairs calibration and validation, thanks to its minimal sensitivity to APS and DEM (in spaceborne case). In particular the MS analysis applied to the TOPSAR acquisition modes of Sentinel-1 (IW ans EW) allows to achieve a millimetric accuracy in the estimation of relative baseline error. According to the analyses performed on a set of Sentinel-1 data the orbit product provided (restituted and precise orbits products) meet the expected accuracy. Eventually we would warn

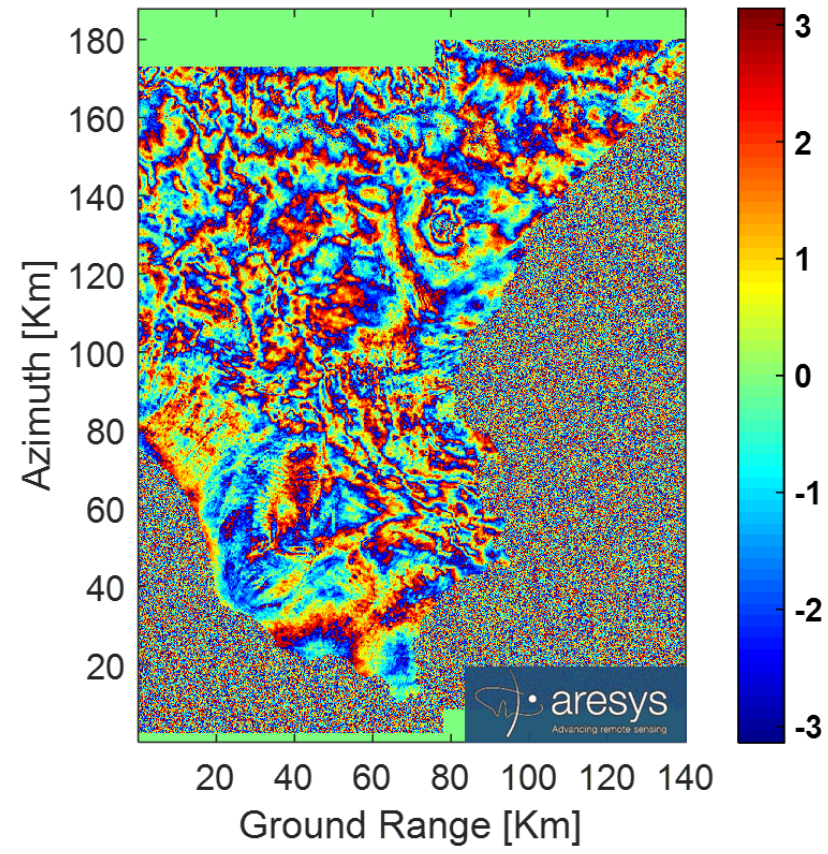

Figure 9: Etna volcano: zoom out of the flattened interferogram, coregistration has been performed by "Precise orbit" product.

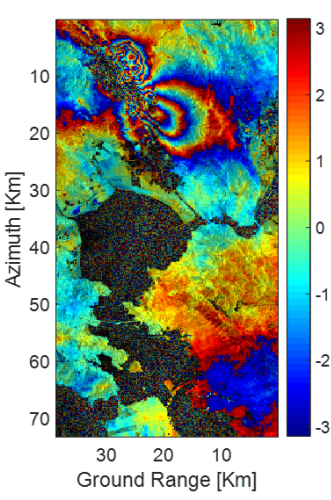

(a)

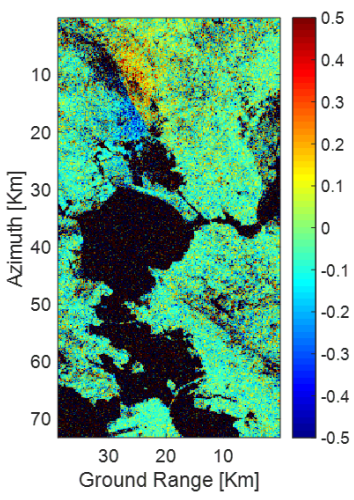

(b)
Figure 10: Napa valley (California): zoom out of the flattened interferogram [rad] (a) and MS interferogram [rad] (b) sensing two components of the same target motion due to an earthquake. For the sake of clearness we reduce the saturation of pixels with low coherence.

the reader against the non-stationary areas, which could alter the results of the technique.

\section{ACKNOWLEDGMENTS}

The authors would like to thank Aresys s.r.l. for the preprocessing of data. 


\section{REFERENCES}

[1] R. Abdelfattah and J.-M. Nicolas. Topographic sar interferometry formulation for high-precision dem generation. Geoscience and Remote Sensing, IEEE Transactions on, 40(11):2415-2426, 2002. ISSN 0196-2892. doi: 10.1109/TGRS.2002.805071.

[2] R. Bamler and M. Eineder. Split band interferometry versus absolute ranging with wideband sar systems. In Geoscience and Remote Sensing Symposium, 2004. IGARSS '04. Proceedings. 2004 IEEE International, volume 2, pages 980-984 vol.2, Sept 2004. doi: 10.1109/IGARSS.2004.1368573.

[3] A. Guarnieri and S. Tebaldini. On the exploitation of target statistics for sar interferometry applications. Geoscience and Remote Sensing, IEEE Transactions on, 46(11):3436 3443, nov. 2008. ISSN 0196-2892. doi: 10.1109/TGRS.2008.2001756.

[4] S. Mancon, A. Monti Guarnieri, S. Tebaldini, and D. Giudici. Orbital error estimation through multisquint analysis. In EUSAR 2014; 10th European Conference on Synthetic Aperture Radar; Proceedings of, pages 1-4. VDE, 2014.

[5] A. Pepe, P. Berardino, M. Bonano, L. Euillades, R. Lanari, and E. Sansosti. Sbas-based satellite orbit correction for the generation of dinsar timeseries: Application to radarsat-1 data. Geoscience and Remote Sensing, IEEE Transactions on, 49(12): $5150-5165$, dec. 2011. ISSN 0196-2892. doi: 10.1109/TGRS.2011.2155069.

[6] P. Prats-Iraola, R. Scheiber, L. Marotti, S. Wollstadt, and A. Reigber. Tops interferometry with terrasarx. Geoscience and Remote Sensing, IEEE Transactions on, 50(8):3179-3188, Aug 2012. ISSN 01962892. doi: 10.1109/TGRS.2011.2178247.

[7] A. Reigber, P. Prats, and J. Mallorqui. Refined estimation of time-varying baseline errors in airborne SAR interferometry. IEEE Geoscience and Remote Sensing Letters, 3(1):145-149, Jan 2006. doi: 10.1109/LGRS.2005.860482.

[8] P. Rosen, S. Hensley, I. Joughin, F. K.Li, S. Madsen, E. Rodriguez, and R. M. Goldstein. Synthetic aperture radar interferometry. Proceedings of the IEEE, 88(3):333-382, 2000. ISSN 0018-9219. doi: $10.1109 / 5.838084$.

[9] R. Scheiber and A. Moreira. Coregistration of interferometric sar images using spectral diversity. Geoscience and Remote Sensing, IEEE Transactions on, 38(5):2179-2191, 2000. ISSN 0196-2892. doi: $10.1109 / 36.868876$.

[10] S. P. Team. Gmes sentinels pod service file format specification. Technical document, European Space Agency (ESA), 2011.

[11] S. P. Team. Gmes sentinels pod service file format specification. 2011. 